\title{
Isolation of Stable, Variously Colored Callus Lines in Portulaca sp. 'Jewel' and Analysis of Betalain Composition
}

\author{
Naonobu NODA and Taiji ADACHI* \\ Applied Genetics and Biotechnology Division, Faculty of Agriculture, Miyazaki University, \\ Miyazaki, 889-2192, Japan \\ * Present address: Department of Plant Science, College of Agriculture, Osaka Prefecture University, \\ 1-1 Gakuen-cho, Sakai, Osaka, 599-8531, Japan. \\ * Corresponding author E-mail address :
}

Received 30 August 1999; accepted 29 November 1999

\begin{abstract}
We have isolated pigmented callus lines in Portulaca sp. 'Jewel' that express distinctly different qualities of color. In this process, yellow and orange lines could be separated from an established magenta callus and stably maintained. A red line was isolated from an orange line. The color of pigmented Portulaca lines is produced through various combinations of betacyanins and betaxanthins. In our pigmented cultures, the main component of betacyanins was betanin while betaxanthins were primarily represented by vulgaxanthin I. The betacyanin content in yellow and orange callus lines was fifty and eight times lower, respectively, than in magenta callus. Suppression of betanin synthesis and the simultaneous increase of vulgaxanthin I accumulation was observed in all yellow and orange callus lines. The variously colored callus lines could be stably maintained on $\mathrm{J} 1$ solidified medium supplemented with $4.5 \mu \mathrm{M} 2,4-\mathrm{D}$ and $30 \mathrm{~g} l^{-1}$ sucrose. At low concentrations of 2,4-D, suppression of betacyanin synthesis in yellow and orange callus could be partially reversed. In orange callus, betacyanin accumulation was also alleviated by treatment with the DNA methylation inhibitor 5azacytidine $(5 \mathrm{AzaC})$. Our Portulaca callus cultures of various pigmentation, combined with the possibility of controlling the colorization, should be useful for studying the gene regulation of the branching process of violet betacyanins and yellow betaxanthins in the betalain biosynthesis pathway.
\end{abstract}

\section{Introduction}

Flower color is the single most important trait in breeding of ornamentals. The major pigments responsible for flower color are the flavonoids, the carotenoids and the betalains. Betalains are pigments that are restricted to the order Caryophyllales and some higher fungi. All betalains contain a betalamic acid moiety which spontaneously condenses with either an amino acid or amine to form yellow betaxanthins or with a cyclo-DOPA derivative to form violet betacyanins (Schliemann et al., 1999; see Fig. 1). In floral tissue of Portulaca grandiflora, the betalain pigments produce yellow, orange, red and magenta variations of color depending on the composition and ratio of yellow betaxanthins and violet betacyanins (Ootani and Hagiwara, 1969). Most betalain producing cell cultures have been shown to accumulate predominantly betacyanins only. An exception is Beta vulgaris (Girod and Zrÿd, 1991; Leathers et al.,
1992) which has been reported to occasionally accumulate betaxanthins and, thus, may produce yellow or orange colored callus. However, different colors did only develop on media with different hormone contents. Yellow, betaxanthin-producing callus was also reported in Portulaca grandiflora (Böhm et al., 1991; Böhm and Böhm, 1996). In this communication, we report the isolation of diverse callus lines displaying yellow, orange and red colorization under identical culture conditions. All these yellowish-red lines were selected from an established magenta-colored callus culture of Portulaca sp. 'Jewel'. The genetic mechanism leading to these fundamental changes in pigment synthesis and accumulation is discussed.

\section{Materials and Methods}

\subsection{Callus culture}

Portulaca sp. 'Jewel' (inbred line JR, red flowering genotype, cf. Adachi, 1972) seedlings in vitro were excised and transferred to MS 


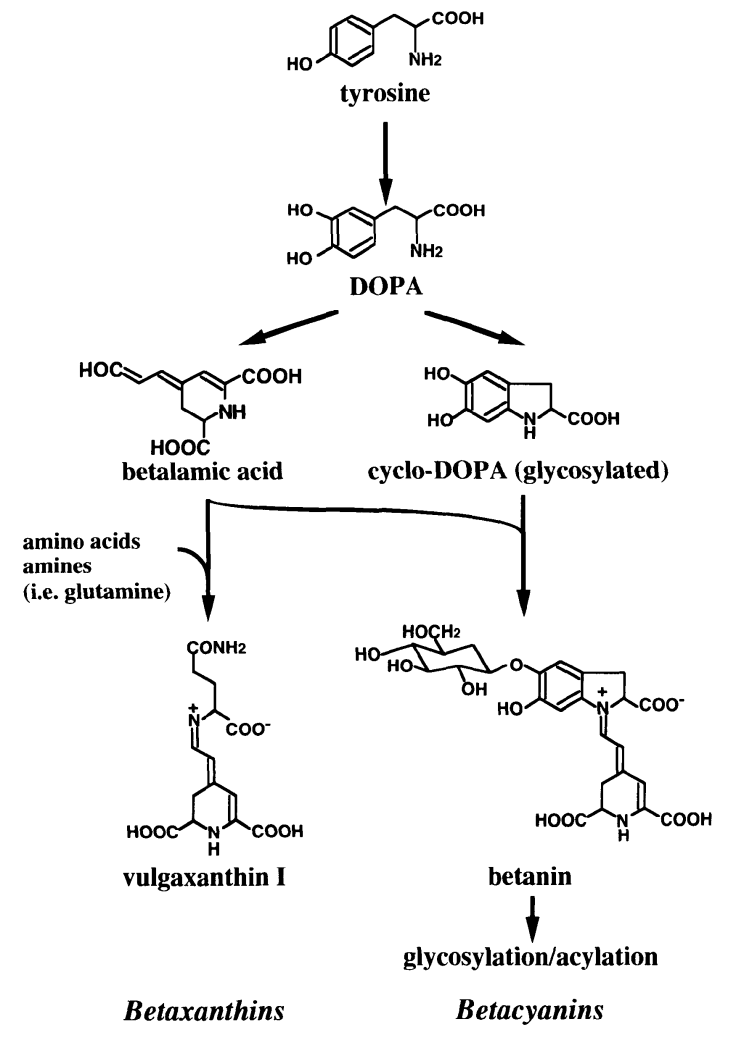

Fig. 1. Biosynthetic pathway of betalain (DOPA: 3,4-dihydroxyphenylalanine).

(Murashige and Skoog, 1962) medium supplemented with $30 \mathrm{~g} \mathrm{l}^{-1}$ sucrose, $9 \mu \mathrm{M}$ 2,4-dichlorophenoxyacetic acid (2,4-D) and $2.5 \mathrm{~g}^{-1}$ gellan gum for the induction of magenta-colored callus (Kishima et al., 1991). These calli maintained high productivity of betacyanins for now 8 to 9 years and stably exhibited purple to magenta pigmentation when selectively subcultured every 25 days. All experiment was performed at $25{ }^{\circ} \mathrm{C}$ under the continuous light condition (photon flux density = $33.3 \mu \mathrm{mol} \mathrm{m} \mathrm{m}^{-2} \mathrm{~s}^{-1}$ ). Yellow and orange callus was spontaneously produced by two magenta-colored lines (JR4 and JR12) five years after initiation. The divergent calli were selectively subcultured every three weeks on MS and J1 (Girod and Zrÿd, 1991) solidified medium. The DNA methylation inhibitor 5 -azacytidine $(5 \mathrm{AzaC})$ was filter sterilized and added to autoclaved medium. Orange callus was cultured on $\mathrm{J} 1$ medium supplemented with $2.25 \mu \mathrm{M}$ 2,4-D and different concentrations of 5AzaC. After 25 days of culture, betalain content of callus was measured.

\subsection{Betalain analysis}

The betalain composition of callus lines displaying visibly different pigmentation was analyzed by HPLC. Betalains were extracted with cold $80 \%$ methanol from frozen calli. The supernatant of extracts was concentrated in vacuo and redissoloved in water. HPLC analysis was performed on a Shimadzu STR ODS-II column $(150 \times 4.6 \mathrm{~mm}$ i. d.) at $35{ }^{\circ} \mathrm{C}$ with a flow rate of $1 \mathrm{ml} \mathrm{min}{ }^{-1}$. The solvent system was $5 \%$ acetic acid (solvent $\mathrm{A}$ ) and $50 \%$ acetonitrile (solvent $\mathrm{B}$ ) with linear gradient elution for $20 \mathrm{~min}$ from 0 to $40 \% \mathrm{~B}$ in A. Betalamic acid, betaxanthins and betacyanins were detected at 430, 480 and $540 \mathrm{~nm}$, respectively. Betaxanthin identification was performed by co-chromatography using semi-synthetic betaxanthins according to Trezzini and Zrÿd (1991) and Toyama et al. (1992). Spectra and absorbances were measured with a Shimadzu multipurpose spectrophotometer (MPS-2000). The concentration of betalains was calculated using the molar extinction coefficient (Girod and Zrÿd, 1991).

\section{Results}

\subsection{Selection and establishment of callus lines with distinctively different pigmentation}

Following the method described by Girod and Zrÿd (1991), betacyanin-producing callus was cultured on $\mathrm{J} 130$ and $5 \mathrm{~J} 110$ medium supplemented with $2,4-\mathrm{D}(0.09,0.225$ and $0.45 \mu \mathrm{M})$ and $6 \mathrm{BAP}$ $(0,0.225$ and $0.45 \mu \mathrm{M})$ in an attempt to initiate cells that mainly accumulate betaxanthin. No such variants were found using this approach. Green calli developed on 6BAP medium, and a low growth rate of callus prevailed on all media tested.

Yellow and orange callus sections were spontaneously formed by a magenta callus line at a low frequency during the latter half of the logarithmic growth phase. These spontaneously induced, initially yellow and orange-colored, mosaic-type calli were grown on $\mathrm{J} 1$ medium supplemented with $4.5 \mu \mathrm{M} 2,4-\mathrm{D}$ and $30 \mathrm{~g}^{-1}$ sucrose and selectively subcultured. The effect of different 2,4-D concentrations $(0.1,1,5,10,50 \mu \mathrm{M})$ on growth and color stability was also examined. Maximum growth rates were observed at 5 or $10 \mu \mathrm{M} 2,4-\mathrm{D}$ on both MS and $\mathrm{J} 1$ medium but growth responses to different 2,4-D concentrations were identical for all isolates independent of their pigmentation, including the magenta mother line (Fig. 2). The maximum content of betacyanin was observed at $0.1 \mu \mathrm{M}$ on the tested concentrations of 2,4-D. However, significant differences in betaxanthin content were not observed (Fig. 3). While the magenta line did not show any change of color in response to different 2,4-D concentrations, the yellow and orange callus produced red cells on 0.1 and $1 \mu \mathrm{M}$ 2,4-D medium (Fig. 4). This result indicates that betacyanin synthesis can be restored at reduced 2,4D. Red callus was also spontaneously produced 


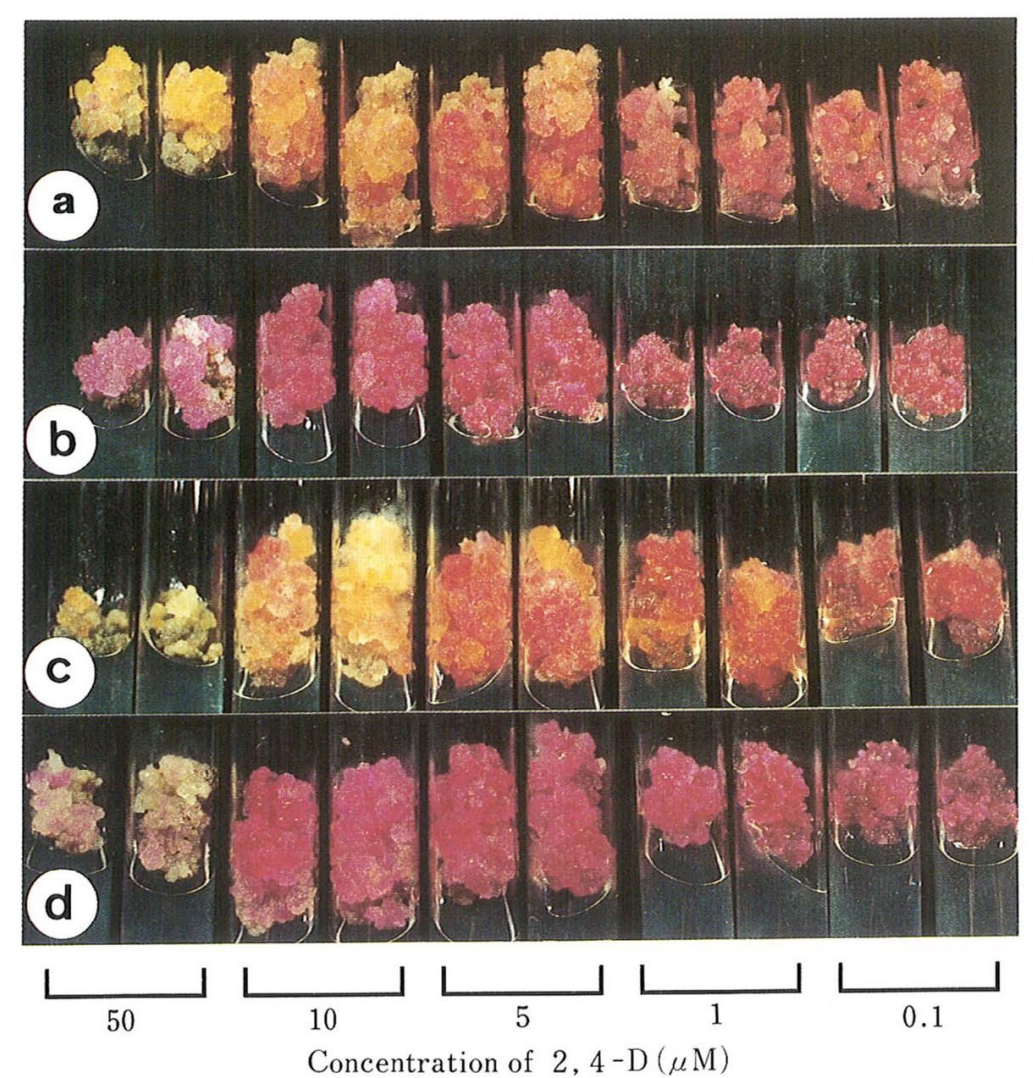

Fig. 4 Portulaca callus cultured on media containing different concentrations of 2,4-D. $\mathrm{a}$ and $\mathrm{b}$ : Callus cultured on J 1 medium, $\mathrm{c}$ and $\mathrm{d}$ : Callus cultured on MS medium, a and $\mathrm{c}$ : Yellow and orange callus derived from magenta JR 4 line on MS medium, b and d: Magenta JR 12 callus line on MS medium.

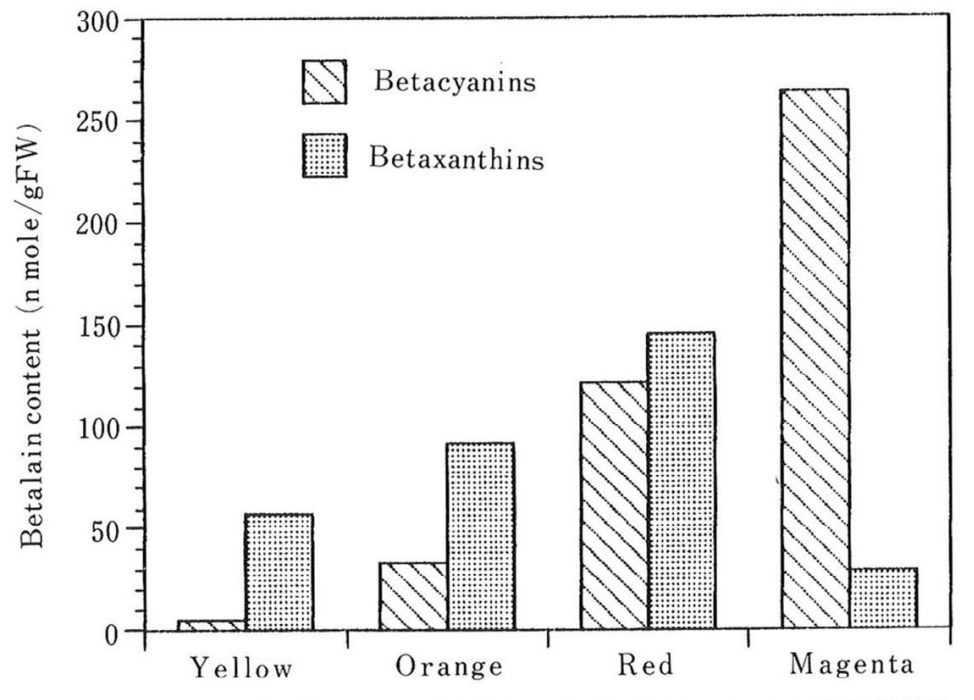

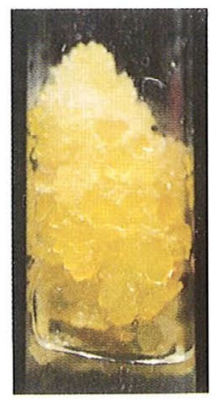

9.0

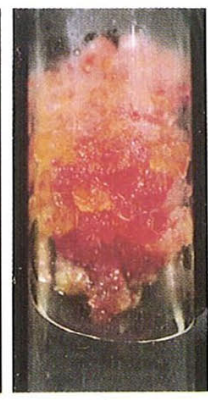

Co

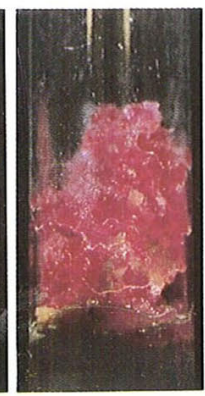

1.5

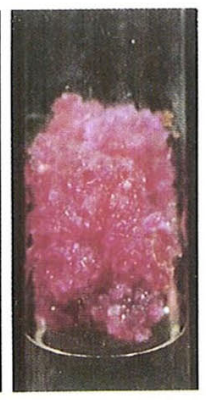

0.4

Fig. 5 Total content of betacyanin and betaxanthin and the betaxanthin/betacyanin $(\mathrm{Bx} / \mathrm{Bc})$ ratio in Portulaca callus lines (JR 4-derived) displaying distinctly different color. 

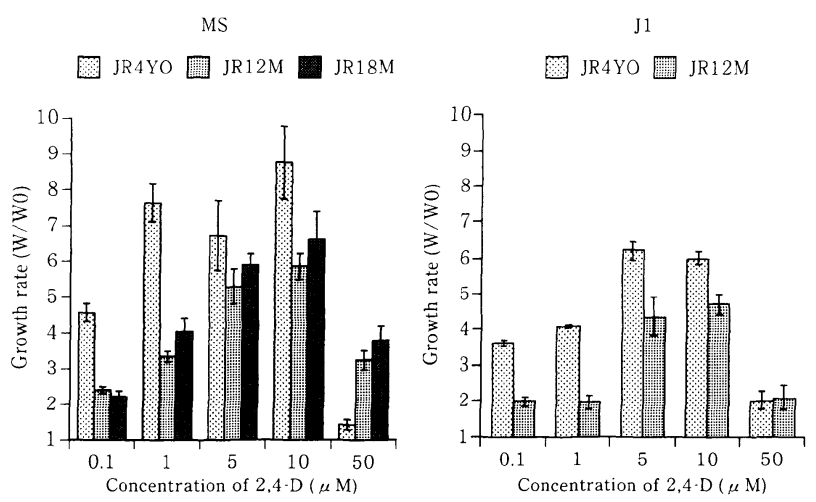

Fig. 2. Effect of different 2,4-D concentrations on growth of pigmented callus lines in Portulaca. Vertical lines indicate SE $(n=10)$. W0 and W denote fresh weights at the start of experiment and sampling times. JR4YO: initial yellow and orange-colored JR4 line, JR12M: magenta colored JR12 line, JR18M: magenta colored JR18 line.
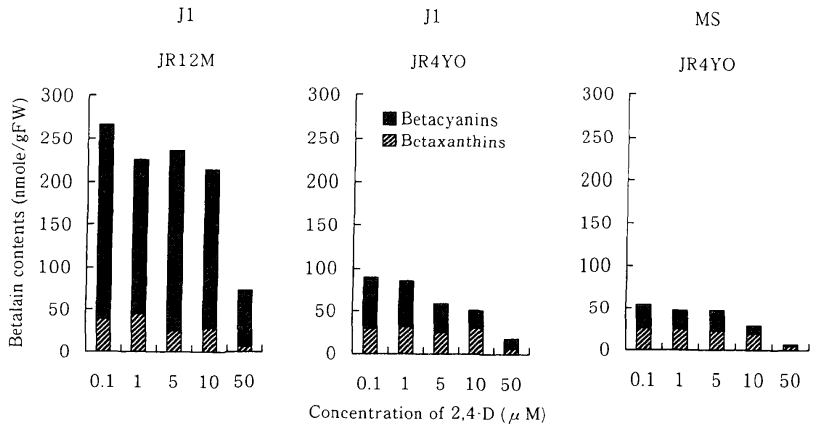

Fig. 3. Effect of different 2,4-D concentrations on betalain accumulation in pigmented callus lines in Portulaca. JR12M: magenta colored JR12 line, JR4YO: initial yellow and orange-colored JR4 line.

from orange callus on $\mathrm{J} 1$ medium. The newly established yellow, orange, red and magenta callus lines became stabilized after repetitive selection and subculture on $\mathrm{J} 1$ medium supplemented with 4.5 $\mu \mathrm{M}$ 2,4-D. Yellow and magenta lines derived from JR4 magenta mother callus on MS medium, were more stabled on J1 medium. Stable yellow and magenta lines were of the friable callus type, while orange and red callus was relatively compact.

\subsection{Characterization of betalain composition in pigmented callus}

As shown in Fig. 5, color of callus can be defined by the betaxanthin/betacyanin $(\mathrm{Bx} / \mathrm{Bc})$ ratio. Betacyanin content in yellow and orange callus was fifty and eight times lower than in magenta callus, respectively. Betaxanthin content in yellow or orange callus was 2 to 3 -fold higher than in magenta callus (Fig. 5). The main components of betacyanins and betaxanthins are betanin and vulgaxanthin $I$, respectively. Yellow and orange callus contained predominantly vulgaxanthin I and only a small amount of betanin (Fig. 6) Red callus, which had spontaneously formed from orange callus, displayed a 7-fold increase of betanin and a 1.5 -fold increase of vulgaxanthin $I$ in comparison to orange callus (Fig. 6). While betalain production was similar in red and magenta callus lines, betaxanthin synthesis was suppressed in magenta callus.

\subsection{Reversion of betacyanin accumulation by me- thylation inhibitor treatment}

As summarized in Fig. 7, callus lines of different color could be isolated from a magenta line that

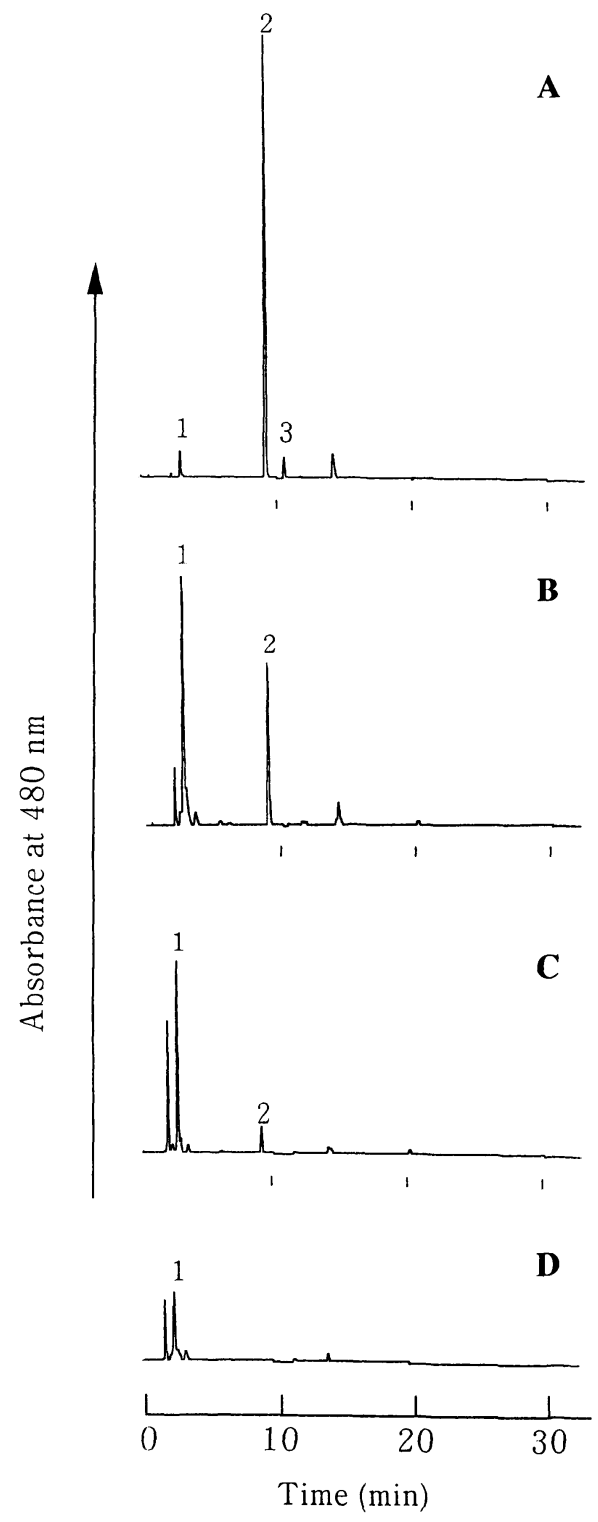

Fig. 6. Chromatogram of methanolic extracts from various colored callus lines (JR4-derived) in Portulaca:. A: magenta colored callus line. B: red colored callus line. $\mathrm{C}$ : orange colored callus line. D: yellow colored callus line. (1) vulgaxanthin I, (2) betanin, (3) isobetanin. 


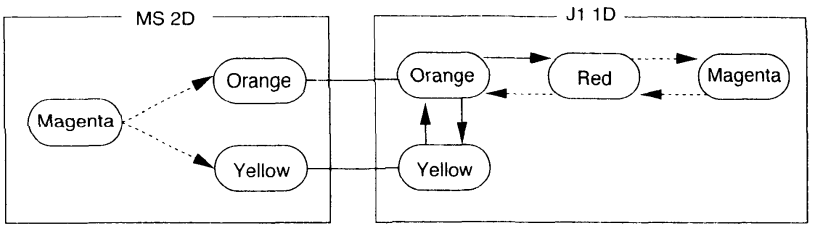

Fig. 7. Spontaneous color changes observed in JR4derived callus lines of Portulaca. Normal arrows indicate low - frequency changes, broken arrows changes that occur at a very low frequency.

(MS 2D = MS medium supplemented with 9 $\mu \mathrm{M} 2,4-\mathrm{D} ; \mathrm{J} 11 \mathrm{D}=\mathrm{J} 1$ medium supplemented with $4.5 \mu \mathrm{M} 2,4-\mathrm{D})$.

produces mainly betacyanin. The mutation in betacyanin synthesis occurred during log-term culture at high concentration of 2,4-D $(9 \mu \mathrm{M})$. Betacyanin accumulation could be recovered on low concentration of 2,4-D medium in mutated (yellow/ orange) callus lines. Progressive methylation can occur during long-term culture. 2,4-D-induced increase of 5-methylcytosine was suggested by Lambé et al. (1997). To confirm the relationship between DNA methylation and betacyanin inhibition, the effect of different $5 \mathrm{AzaC}$ concentrations $(0,5,10,50,100$ and $1000 \mu \mathrm{M})$ on betalain content of freshly grown calli was examined on J1 medium supplemented with 2.25 $\mu \mathrm{M}$ 2,4-D. 5AzaC supplementation did not significantly affect betaxanthin accumulation except at $100 \mu \mathrm{M}$ (Fig. 8). The $\mathrm{Bx} / \mathrm{Bc}$ value was approximately 7.3 at 0 to $10 \mu \mathrm{M} 5 \mathrm{AzaC}$. At concentration higher than $100 \mu \mathrm{M}$, callus became reddish-orange and the $\mathrm{Bx} / \mathrm{Bc}$ value was 3.0. Betacyanin content increased only slightly at 100 and $1000 \mu \mathrm{M} 5 \mathrm{AzaC}$ but the effect on color expression was markedly visible. Betacyanin synthesis suppressed by somatic mutation could be recovered by treatment with DNA methylation inhibitor.

\section{Discussion}

In the present communication, we report the establishment of stable variants of pigmented callus lines in Portulaca sp. 'Jewel'. These variations in color are caused by changes in the ratio of yellow betaxanthins and violet betacyanins. The flower, stem and hypocotyl colors in Portulaca sp. 'Jewel' are devoid of the yellow and orange pigmentation. However, yellow and orange colored callus lines of Portulaca sp. 'Jewel' were spontaneously produced by a magenta mother line through somatic mutations causing suppression of betanin synthesis and an increase of vulgaxanthin I accumulation. In Portulaca grandiflora, yellow colored callus line was produced from red-violet cell culture derived

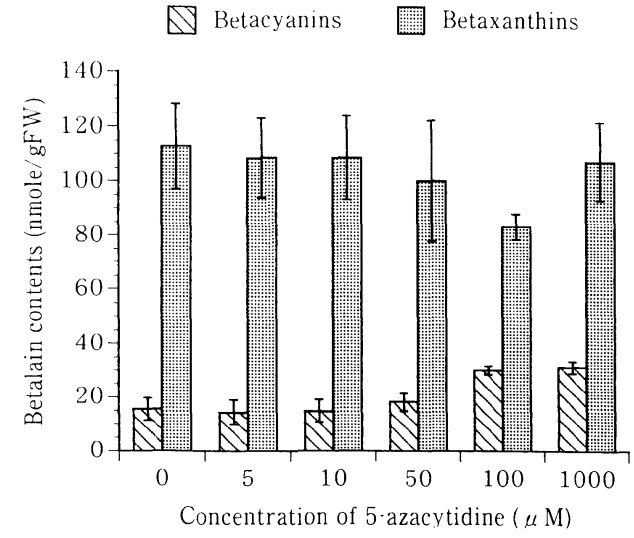

Fig. 8. Effect of 5-azacytidine concentration on betacyanin and betaxanthin accumulation of orange callus line (JR4) in Portulaca. Vertical lines indicate SE $(n=3)$.

from a violet flowering genotype,while not derived from a yellow flowering genotype (Böhm et al., 1991). It seems that cell cultures bring out of the all biosynthetic ability, not depend on original plant genotype.

A possible mechanism for such a metabolic change could be that the inhibition of oxidation of DOPA to cyclo-DOPA resulted in suppression of betanin formation through condensation of cycloDOPA and betalamic acid. Furthermore, free betalamic acid that remained condensed with amino acids may have formed betaxanthins such as vulgaxanthin I (betalamic acid + glutamine). A gene $R$ controlling betacyanin formation was suggested by various authors (Ikeno, 1921; Adachi, 1972; Trezzini and Zrÿd, 1990). It is possible that the expression of $R$ was inhibited by somatic mutation in yellow and orange callus lines. In the red callus line, which was induced spontaneously from orange callus, betacyanin synthesis was recovered and betaxanthin accumulation found to be increased. On the other hand, magenta callus, that was induced spontaneously from red callus as well as the original magenta donor line, showed almost complete suppression of betaxanthin accumulation. This means that a gene responsible for the branching of betacyanin and betaxanthin synthetic pathway must exist. The existence of such a gene was suggested by genetic studies in Portulaca grandiflora. The $B$ (bluing factor) gene changes the red color produced by co-operation of $C$ (chromophore, betalamic acid formation factor) and $R$ gene into magenta (Ikeno, 1921), and the $I$ (inhibitor) gene inhibits or modulates the betaxanthin formation by preventing the conjugation of betalamic acid with amino acids or amines (Trezzini and Zrÿd, 1990). Therefore, diversity of callus color, exhibited as yellow, orange, red and magenta, is associated with $B$ and $I$ 
genes and they probably have the same function.

Changes in color were also observed in stable callus at low frequency. Somatic mutations which affect either $R$ or $I$ genes seem to be unstable. At low concentrations of 2,4-D, suppression of betacyanin synthesis in yellow and orange callus could be partially reversed. When the orange callus line was treated with $5 \mathrm{AzaC}$ at concentrations higher than $100 \mu \mathrm{M}$, the betacyanin content increased and callus color changed to reddishorange. In Beta vulgaris, when cells were cultured on media containing 2,4-D, no color changes by $5 \mathrm{AzaC}$ could be observed (Girod and Zrÿd, 1991). In Portulaca, betacyanin synthesis was also not strongly reactivated by $5 \mathrm{AzaC}$ treatment, because 2,4-D concentarion was still effective. The poly (ADP-ribose) polymerase (PADPRP) is also involved in the suppression of betacyanin synthesis in Beta vulgaris (Girod and Zrÿd, 1991). PADPRP has a function in the induction of defensive metabolism to oxidative stress or mutagens and repair to DNA damages (Satoh and Lindahl, 1992, Berglund et al., 1996). It is possible that PADPRP is required for reactivation of betacyanin synthesis in yellow and orange callus lines of Portulaca. Our result suggests that, in Portulaca callus, the mutated, inactive state of the genes involved in betalain biosynthesis was triggered and/or maintained by hypermethylation of $\mathrm{CpG} / \mathrm{CpNpG}$ sites (Diéguez et al., 1998). Therefore, restoration of betacyanin accumulation at reduced $2,4-\mathrm{D}$ may be induced by demethylation of hypermethylated sites. The hypermethylated state may possibly be habituated by long-term subculture (Lambé et al., 1997). As a result, the different callus lines were uniformly colored and stable.

In conclusion, suppression of betanin synthesis and increase of vulgaxanthin $I$ accumulation was caused by somatic mutation and as a result, the color of callus changed from magenta to yellow or orange. Betacyanin inhibition in yellow callus could be alleviated in the presence of a low concentration of 2,4-D and through treatment with a high concentration of $5 \mathrm{AzaC}$, a DNA methylation inhibitor. All the various colored callus lines could be maintained on $\mathrm{J} 1$ solidified medium supplemented with $4.5 \mu \mathrm{M} 2,4-\mathrm{D}$ and $30 \mathrm{~g} l^{-1}$ sucrose. This callus culture system should be useful for studying gene regulation of the branching process in betacyanin/ betaxanthin biosynthesis.

\section{Acknowledgements}

The authors are grateful to Prof. Franz Hoffmann (University of California, Irvine) and Mr. Arun Nair (Miyazaki University) for critical reading and correction of the manuscript.

\section{References}

Adachi, T., 1972. Chemogenetic studies on flower color in the genus Portulaca in relation to breeding. Bull. Lab. Plant Breeding Fac. Agric. Miyazaki Univ., 3: 1-94. (in Japanese).

Berglund, T., Kalbin, G., Strid, A., Rydstrom, J., Ohlsson A. B., 1996. UV-B- and oxidative stress-induced increase in nicotinamide and trigonelline and inhibition of defensive metabolism induction by poly (ADP-ribose) polymerase inhibitor in plant tissue. FEBS Lett., 380: $188-193$.

Böhm, H., Boehm, L., 1996. Portulaca grandiflora Hook. and $P$. oleracea L.: Formation of betalains and unsaturated fatty acids. In: Y. P. S. Bajaj (Eds.): Medical and Aromatic Plants IX, Biotechnology in Agriculture and Forestry Vol. 37., 335-354. Springer-Verlag, Berlin Heidelberg

Böhm, H., Bohm, L., Rink, E., 1991. Establishment and characterization of a betaxanthin-producing cell culture from Portulaca grandiflora. Plant Cell Tiss. Org. Cult., 26: $75-82$.

Diéguez, M., J., Vaucheret, H., Paszowski, J., Mittelsten Scheid, O., 1998. Cytosine methylation at CG and CNG sites is not a prerequisite for the initiation of transcriptional gene silencing in plants, but it is required for its maintenance. Mol. Gen. Genet., 259: 207-215.

Girod, P.-A., Zryd, J.-P., 1991. Secondary metabolism in cultured red beet (Beta vulgaris L.) cells: Differential regulation of betaxanthin and betacyanin biosynthesis. Plant Cell Tiss. Org. Cult., 25: $1-12$.

Ikeno, S., 1921. Studies on the genetics of flower-colours in Portulaca grandiflora. J. Coll. Agr. Imp. Univ. Tokyo, 8: $93-133$.

Lambé, P., Schie, H., Mutambel, H. S. N., Fouché, J.-G., Deltour, R., Foidart, J.-M., Gaspar, T., 1997. DNA methylation as a key process in regulation of organogenic totipotency and plant neoplastic progression? In Vitro Cell. Dev. Biol.-- Plant, 33: 155 - 162.

Leathers, R. R., Davin, C., Zryd, J.-P., 1992. Betalain producing cell cultures of Beta vulgaris $\mathrm{L}$. var. Bikores Monogerm (red beet). In Vitro Cell. Dev. Biol.-Plant, 28: $39-45$.

Kishima, Y., Nozaki, K., Akashi, R., Adachi, T., 1991. Light - inducible pigmentation in Portulaca callus; selection of a high betalain producing cell line. Plant Cell Rep., 10: $304-307$.

Murashige, T., Skoog, F., 1962. A revised medium for rapid growth and bioassay with tobacco tissue culture. Physiol. Plant., 15: 473-497.

Ootani, S., Hagiwara, T., 1969. Inheritance of flower colors and related chymochromic pigments in $F_{1}$ hybrids of common Portulaca, Portulaca grandiflora. Japan J. Genetics, 44: 65-79.

Satoh, M. S., Lindahl, T., 1992. Role of poly (ADP-ribose) formation in DNA repair. Nature, 356: 356-358.

Schliemann, W., Kobayashi, N., Strack, D., 1999. The decisive step betaxanthin biosynthesis is a spontaneous 
reaction. Plant Physiol., 119: 1217-1232.

Toyama, J., Terahara, N., Adachi, T., 1992. Identification of betalain pigments and the changes of HPLC pattern during developmental stage in some Portulaca genotypes. Breeding Science, 42 (suppl. 2) : 330-331. (in Japanese).

Trezzini, C. F., Zryd, J.-P., 1990. Portulaca grandiflora: A model system for the study of the biochemistry and genetics of betalain synthesis. Acta Horticulturae, 280: 581-585. 